\title{
Effects of repeated lipopolysaccharide treatment on growth performance, immune organ index, and blood parameters of Sprague-Dawley rats
}

\author{
Youbao Zhong ${ }^{1,2}$, Xianlai Zhang ${ }^{1}$, Xiaofen $\mathrm{Hu}^{1}$, Yong $\mathrm{Li}^{1,3}$ \\ ${ }^{1}$ College of Animal Science and Technology, \\ Jiangxi Agricultural University, Nanchang 330045, Jiangxi, China \\ ${ }^{2}$ Experimental Animal Science and Technology Center, \\ Jiangxi University of Traditional Chinese Medicine, Nanchang 330004, Jiangxi, China \\ ${ }^{3}$ Jiangxi Provincial Key Laboratory for Animal Health, College of Animal Science and Technology, \\ Jiangxi Agricultural University, Nanchang 330045, Jiangxi, China \\ liyong2912@163.com
}

Received: May 27, $2018 \quad$ Accepted: September 17, 2018

\begin{abstract}
Introduction: The study was designed to investigate the effects of repeated lipopolysaccharide (LPS) treatment on growth performance, lymphoid organ indexes, and blood cells in Sprague-Dawley rats. Material and Methods: Forty healthy weaned Sprague-Dawley rats were randomly equally divided into LPS and control groups. Each rat in the LPS group was injected via the caudal vein with LPS $(100 \mu \mathrm{g} / \mathrm{kg}$ b.w.) for 10 days, and the control group was treated with an equal volume of normal saline. On the $1^{\text {st }}, 4^{\text {th }}, 7^{\text {th }}$, and $10^{\text {th }}$ days, growth performance, lymphoid organ indexes, and blood cells were evaluated in five necropsied rats. Results: When rats were treated 3-10 times with LPS, their body weight and average daily gains increased more slowly than in the control group $(\mathrm{P}<0.05)$. Repeated LPS treatment significantly increased spleen weight and the ratio of spleen to body weight $(\mathrm{P}<0.05)$. White blood cells, neutrophils, and neutrophil percentage increased $(\mathrm{P}<0.05)$ remarkably, but lymphocyte percentage, haemoglobin, and blood platelet counts decreased significantly $(\mathrm{P}<0.05)$. Conclusion: LPS treatment obviously suppresses growth and promotes peripheral immune organ proliferation. It is indicated that host protective mechanism can be activated by multiple small doses of LPS and prevents organs from further damage during stress status.
\end{abstract}

Keywords: rat, lipopolysaccharide, growth performance, immune organ index, blood cells.

\section{Introduction}

Immune challenge is regarded as a major obstacle to achieving the animal's genetic potential for growth or to efficiency of weight gain (21). Lipopolysaccharide (LPS), an important component of Gram-negative bacterium cytoderm (18), can be used as a stressor to activate the immune system (4). The acyl is inlaid into the outer membrane of bacteria, and the sugar chains are exposed to the outer surface of bacteria (9). Thus, LPS has strong antigenspecificity and can be recognised by antigenpresenting cells. LPS can induce inflammation and a series of immune responses when it is released into the cardiovascular system after the death and lysis of bacteria (22). The effects of LPS on immune function are evidenced in fever, leukocyte reaction, and endotoxaemia. LPS activates mononuclear macrophages to synthesise and release pyretic cytokines indirectly or directly acting on temperature set point, thus leading to abnormal temperature rises (7). As the basic parameters of animal health, blood analysis is quite useful to diagnose their diseases (11, 17). White blood cells help the body to resist invasions of pathogenic microorganisms, and an animal's body will recruit a large number of neutrophils into infection sites during acute inflammation. After LPS enters a host, the activated 
neutral cytokines would stimulate marrow to release plenty of neutrophils into the circulatory system, resulting in small molecular antigens being swallowed by neutrophils (15). As the core of specific immunity, lymphocytes are responsible for both humoral and cellular immunity. When animals have suffered severe trauma or have acquired infection, bacteria from blood and lesions release excessive LPS into the circulation system. Subsequently, this causes septic shock and endotoxaemia (26).

An LPS-treated animal model is more standardised and reproducible, less expensive to develop, and more suitable to access than a bacterial infection model $(10,13)$. Nowadays, LPS is widely applied in research on Gram-negative bacterial infections and sepsis in various animal stress models $(19,20,22)$. As we know, weaned domestic animals are easily subjected to various pathogenic bacteria; the changes in immune parameters usually become an important basis upon which to evaluate disease resistance. In this study, the present model was established to investigate effects on growth performance, immune organ index, and blood cells of weaned Sprague-Dawley (SD) rats. It is expected to provide detailed evidence for elucidating the development and reproduction of weaned domestic animals during continuous infection status.

\section{Material and Methods}

Experimental animals. Forty healthy weaned SD rats aged 26 days and weighing $83 \pm 4 \mathrm{~g}$ were kept in the animal anatomy laboratory of Jiangxi Agricultural University. The rats had unrestricted access to feed and water and were fed for three days to relieve the stress reaction prior to the experiment. The rats were randomly equally divided into LPS and control groups. In the LPS group, the rats received a single daily injection of LPS $(100 \mu \mathrm{g} / \mathrm{kg} \mathrm{b.w.)} \mathrm{for} 10$ consecutive days. In the control group, rats were also treated once per day with equal volume normal saline
(NS) via the caudal vein. On the $1^{\text {st }}$ (at 29 days old), $4^{\text {th }}$ (at 32 days old), $7^{\text {th }}$ (at 35 days old), and $10^{\text {th }}$ (at 38 days old) day, five rats from each group were necropsied in $6 \mathrm{~h}$ after the last LPS or NS injection, and blood, the spleen, and the thymus were collected.

Determination of growth performance, blood parameters, and lymphoid organ indexes. On the $1^{\text {st }}, 4^{\text {th }}, 7^{\text {th }}$, and $10^{\text {th }}$ day, body (BW), spleen ( $\left.\mathrm{SW}\right)$, and thymus (TW) weights of each necropsied rat were measured, and the ratio of thymus to body weight (TBR) and the ratio of spleen to body weight (SBR) were calculated. During days 1-4, 4-7, and 7-10, average daily gain (ADG) was calculated as well. In addition, blood parameters, including white blood cells (WBC), lymphocytes (LYM), neutrophils (GRAN), lymphocyte percentage (LYM\%), neutrophil percentage (GRAN\%), haemoglobin (HGB), red blood cells (RBC), and blood platelets (PLT), were examined.

Statistical analysis. SPSS13.0 software (IBM Corporation, USA) was used to perform one-way analysis of variance, and intergroup differences were analysed. All experimental data were tested by analysis of variance with significant differences between means determined by Duncan post-hoc tests.

\section{Results}

Effects of repeated LPS treatment on the growth performance of SD rats. When SD rats received a single-dose injection of LPS (100 $\mu \mathrm{g} / \mathrm{kg} \mathrm{b.w.)}$ for 4,7 , and 10 consecutive days, their body weights were lower than in control rats $(\mathrm{P}<0.05$, $\mathrm{P}<0.01$, and $\mathrm{P}<0.01$, respectively) (Fig. 1A). The ADG of the LPS group between the $4^{\text {th }}$ and $7^{\text {th }}$ days was significantly lower than that of the control group ( $\mathrm{P}<0.01)$; however, on the $10^{\text {th }}$ day of the experiment, there was no significant ADG difference between the LPS and control groups $(\mathrm{P}>0.05)$ (Fig. 1B).
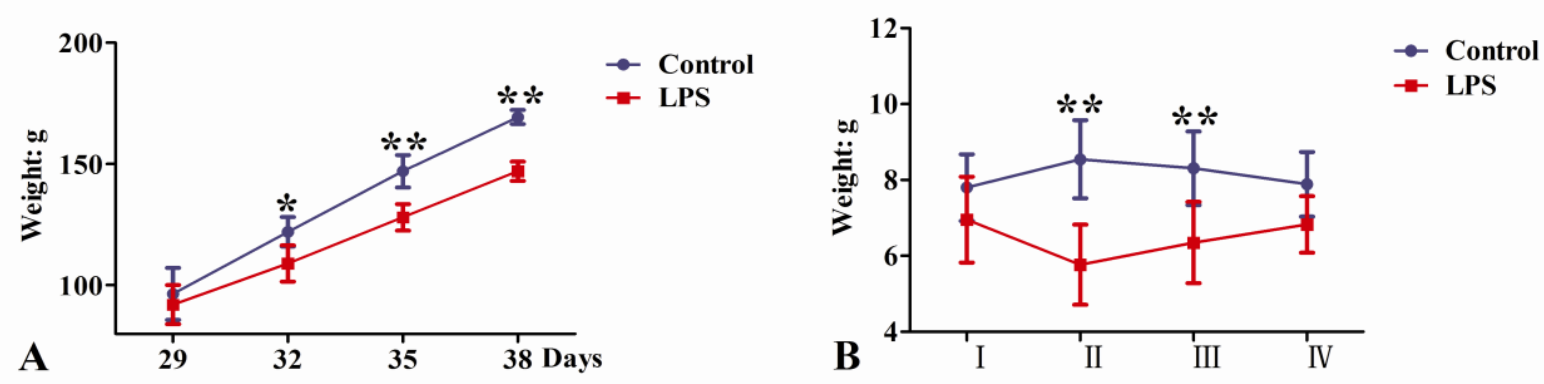

Fig. 1. Effects of LPS treatments on the growth performance of SD rats (* $\mathrm{P}<0.05, * * \mathrm{P}<0.01)$. A - body weight, B $-\mathrm{ADG}, \mathrm{I}-$ between days 26 and 29, II - between days 29 and 32, III - between days 32 and 35, IV - between days 35 and 38 
Effects of repeated LPS treatments on lymphoid organ indexes of SD rats. Both the SW and SBR of 29-, 32-, 35-, and 38-day-old rats in the LPS group were notably higher than in the control group ( $\mathrm{P}<0.05$ ) (Fig. 2A, C). On days 29, 32, and 35, there were no obvious TW and TBR differences between LPS- and NS-treated rats $(\mathrm{P}>0.05)$ (Fig. 2B, D). The TW of the LPS group was significantly lower in 38-day-old rats than in the control group $(\mathrm{P}<0.05)$, but without there being significant TBR difference one from the other $(\mathrm{P}>0.05)$ (Fig. 2B, D).

Effects of repeated LPS treatments on blood parameters of SD rats. For rats of the same age in days, the WBCs of the LPS group were remarkably higher than those of the control group $(\mathrm{P}<0.05)$. In the LPS group, the WBCs of 32-, 35-, and 38-dayold rats were significantly higher than those of 29day-old rats $(\mathrm{P}<0.05)$ (Fig. 3A). However, no significant RBC difference was found between the LPS and control groups on the same day of the rats' lives $(\mathrm{P}>0.05)$, and no obvious $\mathrm{RBC}$ change was found among rats of differing ages in days $(P>0.05)$ (Fig. 3B). The LYM of the LPS group was pronouncedly lower than that of the control group only for 32-day-old rats $(\mathrm{P}<0.05)$ (Fig. 3C). However, compared with rats of the same age in days from the control group, the LYM\% of LPS-treated rats decreased notably $(\mathrm{P}<0.05)$. For the LPS group, the LYM\% of 32-day-old rats was obviously lower than those of 29-, 35-, and 38-day-old rats $(\mathrm{P}<0.05)$ (Fig. 3D). For 29-, 32-, 35-, and 38-day-old rats, the GRAN and GRAN\% of LPS group were higher than those of the control group $(\mathrm{P}<0.05)$ (Fig. 3E, F). The GRAN\% of 32- and 35-day-old LPS-treated rats were higher compared to 38-day-old LPS-treated rats $(\mathrm{P}<0.05)$ (Fig. 3F). A significantly lower HGB pertained to the LPS group than to the control group on the same day of life $(\mathrm{P}<0.05)$ (Fig. 3G), and the PLT of LPS-treated rats were vastly lower than those of the control group $(\mathrm{P}<0.05)$ (Fig. 3H).
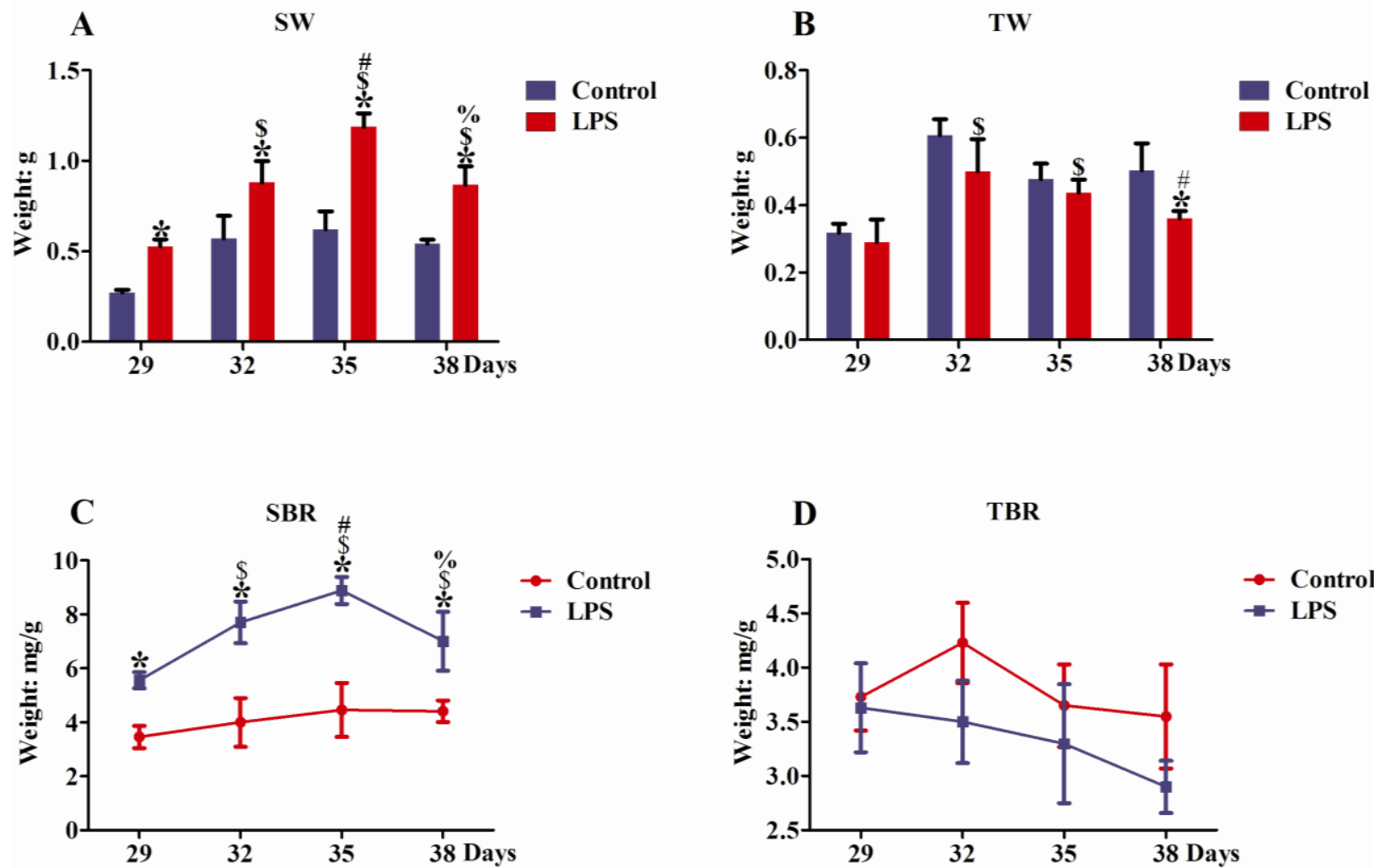

Fig. 2. Effects of LPS treatments on lymphoid organ indexes of SD rats. A - spleen weight, B - thymus weight, C - the ratio of spleen to body, $\mathrm{D}-$ the ratio of thymus to body. The following symbols indicates significant changes in lymphoid organ indexes $(\mathrm{P}<0.05): *$ spleen and thymus differences between LPS and control group on the same days, \$ - LPS treatment for day 29 versus LPS treatment for days 32 , 35, and 38, \# - LPS treatment for day 32 versus LPS treatment for days 35 and 38, \% - LPS treatment for day 35 versus LPS treatment for day 38 

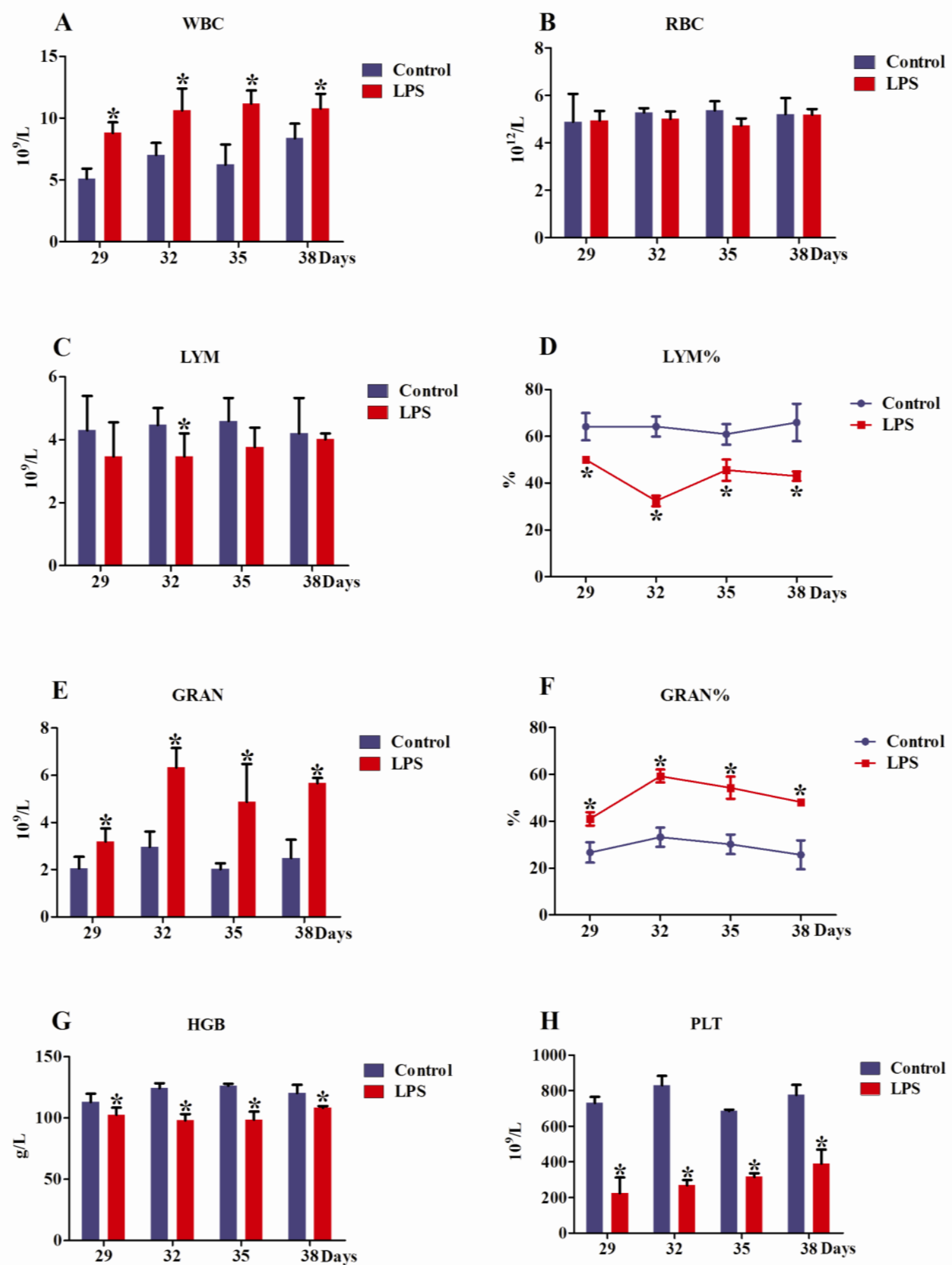

Fig. 3. Effects of LPS treatments on blood parameters of SD rats. A - white blood cells, B - red blood cells, C - lymphocytes, $\mathrm{D}$ - lymphocyte percentage, $\mathrm{E}$ - neutrophils, $\mathrm{F}$ - neutrophil percentage, $\mathrm{G}$ - haemoglobin, $\mathrm{H}$ - platelets. * means the same as in Fig. 2

\section{Discussion}

LPS challenge negatively affects SD rat digestive function. Recently, it has been reported that the LPS challenge activated immune responses and negatively impacted the growth performance of domestic animals $(1,25)$. In present studies, the growth rate of SD rats was obviously inhibited after multiple small dose LPS injections so that dysplasia appeared in the rats. When LPS directly enters the cardiovascular 
system via the caudal vein, it raises concentrations of pro-inflammatory cytokines (IL-1 $\beta$, IL-6, and TNF- $\alpha$ ) in the blood (24), and then these cytokines feed information back to the central nervous system and suppress the animal's appetite (6). In addition, LPS can inhibit the synthesis and secretion of thyroid hormone, thus leading to weaker peristalsis of gastrointestinal motility and intestinal digestion and decreased absorption ability (1).

LPS challenge activated the protective changes in SD rats. The weight and related indexes of animal immune organs are indispensable in evaluating the speed of growth and condition of the immune system (2). This study demonstrated that the SW and SBR of LPS-treated rats increased significantly. As the biggest peripheral immune organ, the spleen can initiate immune response to a haematogenous antigen. Accordingly, LPS can spur excessive lymphocyte proliferation and induce large-scale pro-inflammatory cytokines production (12), thus resulting in splenic enlargement. Surprisingly, the SW and SBR of 38-dayold LPS-treated rats were manifestly lower than those of 35-day-old LPS-treated rats. Multiple injections of small doses of LPS induce the host's protective mechanism and may help the host to resist LPS stimulation again and protect the spleen from further damage. Although the TW and TBR of the rats decreased, a significant difference was not found between the LPS and control groups except for in the TW of 38-day-old rats. This result is inconsistent with the reported result of Huang et al. (5). It is indicated that external LPS stimulation more easily effects protective changes for the animal's spleen than the thymus.

Blood is the essential component of various animals and takes on many physiological functions. Its physical and chemical properties are relatively stable; conversely, the alteration of blood cells and the emergence of abnormal material not only embody the physiological and pathological changes in circulation system, but also present the pathologic changes of related organs (23). When an animal is infected with bacteria, leukocytes directionally migrate into inflammatory sites and secrete a large number of chemokines, adhesion factor, and pro-inflammatory cytokines to eliminate corresponding pathogens in a coordinated way (3). As the present study shows, repeated LPS treatment leads to a significant increase in $\mathrm{WBC}$ counts in rats. A high number of WBCs is quite useful for the host to resist bacteria invasion. Lymphocytes can participate indirectly or directly in specific immune responses, and their percentage is an important indicator of immune status. Neutrophilic granulocytes and their percentages are closely related to inflammation and malnutrition (16). In the present results, multiple low-dose LPS injections cause significant reduction of lymphocyte percentage, but only the lymphocyte level of 32-day-old LPS-treated rats was remarkably lower. Neutrophilic granulocytes are called small macrophages and could eliminate bacteria, tissue fragments, and exogenous protein in the early stages of inflammation. Therefore, an increased number of neutrophilic granulocytes are helpful to maintain the body's homeostasis. Higher concentrations of PLT can enhance the stress resilience of the host and accelerate the rebuilding rate of injured blood vessels. During LPS treatment, the significant PLT decrease would affect the resistance of rats to various pathogens and inhibit wound healing. Similarly, LPS induces production of IgG with expression of B-cell immune response-related cell surface molecules and also stimulates activation of T-lymphocytes in peripheral blood mononuclear cells (PBMC) (8). However, ex vivo secretion of IL-6 and TNF- $\alpha$ by porcine PBMC can be modulated by some drugs after LPS stimulation (14). In brief, the balance change in blood parameters protects animals from further damage during the stress induced by LPS.

In conclusion, the growth and development of SD rats were obviously inhibited, but the spleen was enlarged. The protective changes or response were probably activated by multiple low-dose LPS treatment, and the present study provides some reasonable evidence in the scientific clarification of the stress response mechanism of the rat.

Conflict of Interests Statement: The authors declare that there is no conflict of interests regarding the publication of this article.

Financial Disclosure Statement: This study was supported by the National Natural Science Foundation Project of China (No. 31360592), the Science and Technology Project of Jiangxi Province Education Department (No. GJJ13288), and the Natural Science Foundation Project of Jiangxi Province (20181BAB204016).

Animal Rights Statement: The experiment was conducted in accordance with the rules and regulation on animal experimentation of Jiangxi Agricultural University.

Acknowledgements: Youbao Zhong and Xianlai Zhang made equal contributions to this study. Yong Li (liyong2912@163.com )was the corresponding author.

\section{References}

1. Campos P.H., Merlot E., Damon M., Noblet J., Le Floc'h N.: High ambient temperature alleviates the inflammatory response and growth depression in pigs challenged with Escherichia coli lipopolysaccharide. Vet J 2014, 200, 404-409.

2. Cesta M.F.: Normal structure, function, and histology of the spleen. Toxicol Pathol 2006, 34, 455-465.

3. Faas M.M., Moes H., van der Schaaf G., de Leij L.F., Heineman M.J.: Total white blood cell counts and LPS-induced TNF alpha production by monocytes of pregnant, pseudopregnant, and cyclic rats. J Reprod Immunol 2003, 59, 39-52. 
4. Hayley S., Mangano E., Strickland M., Anisman H.: Lipopolysaccharide and a social stressor influence behaviour, corticosterone, and cytokine levels: divergent actions in cyclooxygenase-2 deficient mice and wild type controls. J Neuroimmunol 2008, 197, 29-36.

5. Huang H., Liu A., Wu H., Ansari A.R., Wang J., Huang X., Zhao X., Peng K., Zhong J., Liu H.: Transcriptome analysis indicated that Salmonella lipopolysaccharide-induced thymocyte death and thymic atrophy were related to TLR4-FOS/JUN pathway in chicks. BMC Genomics 2016, 17, 322.

6. Johnson R.W.: Inhibition of growth by pro-inflammatory cytokines: an integrated view. J Anim Sci 1997, 75, 1244-1255.

7. Kaplanski J., Fraifeld V., Rubin M.: Body temperature and hypothalamic PGE2 response to LPS in developing rats. Ann N Y Acad Sci 1997, 813, 474-479.

8. Kim M.H., Yun C.H., Kim G.R., Kol J.Y., Lee J.J., Ha J.K.: Changes of immunoglobulins and lymphocyte subpopulations in peripheral blood from Holstein calves challenged with Escherichia coli lipopolysaccharide. Asian Austral J Anim 2011, 24, 696-706.

9. Lukasiewicz J., Lugowski C.: Biologic activity of lipopolysaccharides. Postepy Hig Med Dosw 2003, 57, 33-53.

10. Myers M.J., Farrell D.E., Palmer D.C., Post L.O.: Inflammatory mediator production in swine following endotoxin challenge with or without co-administration of dexamethasone. Int Immunopharmacol 2003, 3, 571-579.

11. Nishikawa T., Omura M., Kawaguchi M., Takatsu A., Satoh F., Ito S., Kurihara I., Itoh H., Yanase T., Shibata H., Oki Y., Naruse M., Sakurai K., Sasamoto H., Kuwa K.: Calibration and evaluation of routine methods by serum certified reference material for aldosterone measurement in blood. Endocrinol $\mathrm{J}$ 2016, 63, 1065-1080.

12. Obernikhin S.S., Yaglova N.V.: Morphological and functional changes in the thymus and spleen of mouse offspring in the development of systemic inflammatory response after a single immunity stimulation in early pregnancy. Bull Exp Biol Med 2014, 157, 812-815.

13. Olson N.C., Hellyer P.W., Dodam J.R.: Mediators and vascular effects in response to endotoxin. Br Vet J 1995, 151, 489-522.

14. Pomorska-Mól M., Czyżewska-Dors E., Kwit K., Pejsak Z.: Enrofloxacin in therapeutic doses alters cytokine production by porcine PBMCs induced by lipopolysaccharide. Drug Chem Toxicol 2017, 40, 295-299.

15. Qazi M.R., Bogdanska J., Butenhoff J.L., Nelson B.D., DePierre J.W., Abedi-Valugerdi M.: High-dose, short-term exposure of mice to perfluorooctanesulfonate (PFOS) or perfluorooctanoate (PFOA) affects the number of circulating neutrophils differently, but enhances the inflammatory responses of macrophages to lipopolysaccharide in a similar fashion. Toxicology 2009, 262, 207-214.

16. Reddan D.N., Klassen P.S., Szczech L.A., Coladonato J.A., O'Shea S., Owen W.J., Lowrie E.G.: White blood cells as a novel mortality predictor in haemodialysis patients. Nephrol Dial Transplant 2003, 18, 1167-1173.

17. Revathy N.S.: Routine measurements of cord arterial blood lactate levels in infants delivering at term and prediction of neonatal outcome. Med J Malaysia 2016, 71, 131-133.

18. Ronco C.: Lipopolysaccharide from the cellular wall of Gramnegative bacteria, also known as endotoxin, is a key molecule in the pathogenesis of sepsis and septic shock. Blood Purificat $2014,37,1$.

19. Schmidhammer R., Wassermann E., Germann P., Redl H., Ullrich R.: Infusion of increasing doses of endotoxin induces progressive acute lung injury but prevents early pulmonary hypertension in pigs. Shock 2006, 25, 389-394.

20. Seydel U., Hawkins L., Schromm A.B., Heine H., Scheel O., Koch M.H., Brandenburg K.: The generalized endotoxic principle. Eur J Immunol 2003, 33, 1586-1592.

21. Spurlock M.E.: Regulation of metabolism and growth during immune challenge: an overview of cytokine function. J Anim Sci 1997, 75, 1773-1783.

22. Tesh V.L., Morrison D.C.: The physical-chemical characterization and biologic activity of serum released lipopolysaccharides. J Immunol 1988, 141, 3523-3531.

23. Van Balveren J.A., Huijskens M.J., Gemen E.F., Pequeriaux N.C., Kusters R.: Effects of time and temperature on 48 routine chemistry, haematology, and coagulation analytes in whole blood samples. Ann Clin Biochem 2016, 54, 448-462.

24. Webel D.M., Finck B.N., Baker D.H., Johnson R.W.: Time course of increased plasma cytokines, cortisol, and urea nitrogen in pigs following intraperitoneal injection of lipopolysaccharide. J Anim Sci 1997, 75, 1514-1520.

25. Zhang N., Li H., Jiang C., Tu Y., Diao Q.: Effects of lipopolysaccharide on the growth performance, nitrogen metabolism and immunity in preruminant calves. Indian J Anim Res 2017, 51, 717-721.

26. Zhao L., Chen Y.H., Wang H., Ji Y.L., Ning H., Wang S.F., Zhang C., Lu J.W., Duan Z.H., Xu D.X.: Reactive oxygen species contribute to lipopolysaccharide-induced teratogenesis in mice. Toxicol Sci 2008, 103, 149-157. 